\title{
Patient specific respiratory motion modeling using a limited number of 3D lung CT images
}

\author{
Xueli Cui ${ }^{\mathrm{a}, \mathrm{b}, \mathrm{c}}$, Xin Gao ${ }^{\mathrm{a},{ }^{*}}$, Wei Xia ${ }^{\mathrm{a}}$, Yangchuan Liu ${ }^{\mathrm{a}}$ and Zhiyuan Liang ${ }^{\mathrm{d}, *}$ \\ ${ }^{a}$ Medical Imaging Department, Suzhou Institute of Biomedical Engineering and Technology, Chinese \\ Academy of Sciences, Suzhou, Jiangsu 215163, China \\ ${ }^{b}$ Changchun Institute of Optics, Fine Mechanics and Physics, Chinese Academy of Sciences, \\ Changchun, Jilin 130000, China \\ ${ }^{c}$ University of Chinese Academy of Sciences, Beijing 100049, China \\ ${ }^{d}$ School of Biomedical Engineering, Capital Medical University, Beijing 100069, China
}

\begin{abstract}
To build a patient specific respiratory motion model with a low dose, a novel method was proposed that uses a limited number of 3D lung CT volumes with an external respiratory signal. 4D lung CT volumes were acquired for patients with in vitro labeling on the upper abdominal surface. Meanwhile, 3D coordinates of in vitro labeling were measured as external respiratory signals. A sequential correspondence between the 4D lung CT and the external respiratory signal was built using the distance correlation method, and a 3D displacement for every registration control point in the CT volumes with respect to time can be obtained by the 4D lung CT deformable registration. A temporal fitting was performed for every registration control point displacements and an external respiratory signal in the anterior-posterior direction respectively to draw their fitting curves. Finally, a linear regression was used to fit the corresponding samples of the control point displacement fitting curves and the external respiratory signal fitting curve to finish the pulmonary respiration modeling. Compared to a B-spline-based method using the respiratory signal phase, the proposed method is highly advantageous as it offers comparable modeling accuracy and target modeling error (TME); while at the same time, the proposed method requires $70 \%$ less 3D lung CTs. When using a similar amount of 3D lung CT data, the mean of the proposed method's TME is smaller than the mean of the PCA (principle component analysis)-based methods' TMEs. The results indicate that the proposed method is successful in striking a balance between modeling accuracy and number of 3D lung CT volumes.
\end{abstract}

Keywords: Motion model, 4D lung CT, external respiratory signal, deformable registration, temporal fitting

\footnotetext{
*Corresponding authors: Xin Gao, Medical Imaging Department, Suzhou Institute of Biomedical Engineering and Technology, Chinese Academy of Sciences, Suzhou, Jiangsu 215163, China. Tel.: +86 512-69588055; Fax: +86 51269588140; E-mail: xingaosam@yahoo.com.

Zhiyuan Liang, School of Biomedical Engineering, Capital Medical University, Beijing 100069, China. Tel.: +86 1083911681; Fax: +86 10-83911560; E-mail: zhiyl@126.com.
} 


\section{Introduction}

Respiratory motion is a critical aspect behind inaccurate lesion localizations in surgery path planning and surgical navigation. Therefore, accurately modeling respiratory motion is crucial for better estimating and predicting the organ's motion so as to improve the speed and accuracy of lesion localization [1-3].

Different respiratory motion models have been proposed to estimate and predict the motion trends of either gross tumor volume (GTV) or the whole lung. Most of these models, however, require a 4D lung $\mathrm{CT}$ and respiratory signals.

Different means of converting physical measurements into respiratory signals for respiratory motion models have been adopted. Such measurements primarily require that they have a strong relationship with the true motion to be estimated and that they can be acquired relatively easily. Some methods have obtained respiratory signals by implanting and then tracking one or more small gold marks in the target organ or lesion's vicinity [4,5]. While the accuracy of marker tracking is clinically sufficient, the implantation procedure is invasive, and if performed improperly, may cause serious side effects, such as pneumothorax. However, some methods have obtained simpler respiratory signals by deriving them from higher dimensional data such as a 4D lung CT. These respiratory signals have a relatively low temporal resolution [6,7]. Furthermore, a method of acquiring respiratory signals by tracking the motion of one or more points on the surface of the chest or abdomen was widely adopted and can be done using optical tracking technology. Additionally, these external respiratory signals are particularly appealing because they are noninvasive, non-ionizing, low-cost, and can be acquired conveniently $[2,8,9]$.

The respiratory motion models also require series of CT data. The 4D lung CT was usually acquired in cine mode due to the limitations associated with CT technology [7,10-14]. In the process, in order to cover the whole lung area, 25, 30, or even more volumes per couch position were needed, and 3 or 4 contiguous couch positions were scanned for each patient [6,7]. This increases the patient's radiation dose, which can lead to a risk of genetic defects. However, as imaging technology has recently undergone rapid development, the CT scanner can implement dynamic imaging of the whole-lung under free breathing.

Therefore, the main objective of this study was to take advantage of this new imaging technology so as to acquire a limited number of 3D lung CT images in order to build a patient specific respiratory motion model with a low dose in combination with an external respiratory signal. First, the 4D lung CT that were used in the study were acquired on a new generation of CT scanner while the 3D coordinates of in vitro labeling are measured as an external respiratory signal. Furthermore, a distance correlation method was used to build the sequential correspondence between the 4D lung CT and external respiratory signal. Subsequently, 4D lung CT deformable registration was performed to acquire the $3 \mathrm{D}$ displacements for every registration control point with respect to time. The temporal fitting was adopted to obtain both the external respiratory signal fitting curve and the control point displacements fitting curves. Finally, the motion model was constructed by fitting a linear function that related each control point displacement to the corresponding respiratory signal value. 


\section{Materials and methods}

\subsection{Data acquisition}

The 4D lung CT volumes that were used for the study were acquired on a Toshiba Aquilion ONE 320-slice CT scanner using the 'Lung mass perfusion' protocol at the Wuxi No.2 People's Hospital, China. The external respiratory signal was acquired by using a self-developed binocular visual system composed of an infrared LED, computer, and two stereo infrared-detecting cameras.

A $5 \mathrm{~mm}$ straw hat infrared LED with a $120^{\circ}$ projection angle was attached to a patient's upper abdominal surface as in vitro labeling. The binocular visual system must first be switched on in order to determine the sequential correspondence between the 4D lung CT volumes and the external respiratory signal. The 3D coordinates of the labeling, denoted as $D=\left\{D_{j} \mid j=1,2, \ldots, n\right\}$, $D_{j}=\left(D_{j x}, D_{j y}, D_{j z}\right)$, were measured 30 times per second. Ten 3D lung CT volumes $I=\left\{I_{i} \mid i=1,2, \ldots, 10\right\}$ of the patient under deep free breathing was continuously acquired at 1.5 second intervals $(\Delta t)$ using a volume of $512 \times 512 \times 320$ with voxel sizes of $0.677 \times 0.677 \times 0.50 \mathrm{~mm}^{3}$. Furthermore, the obtained CT volume that corresponds to the full expiration was used as the reference volume. Three patient datasets were used in this study, all of which had a 4D lung CT acquisition under deep free breathing.

\subsection{Distance correlation method}

From the binocular visual system's sampling rate as well as from the CT scanner, it can be determined that the elapsed time of recording 46 coordinates (45 intervals) of the labeling by the binocular visual system is equal to that of obtaining two adjacent $3 \mathrm{D}$ lung CT volumes. A distance correlation approach was proposed to construct the sequential correspondence between the 4D lung CT $I$ and the measured 3D coordinates of the labeling $D$ by calculating the distance measure equation. The distance measure equation $F$ was defined as:

$$
F=\min _{1 \leq j \leq n-9 \times 45} \sum_{i=1}^{9}\left|\sqrt{\sum_{r=1}^{3}\left(D_{(j+i \times 45) r}-D_{[j+(i-1) \times 45] r}\right)^{2}}-\sqrt{\sum_{r=1}^{3}\left[q_{r}\left(C_{(i+1) r}-C_{i r}\right)\right]^{2}}\right|
$$

where $q_{r}(r=1,2,3)$ signifies the voxel sizes corresponding to the $\mathrm{x}, \mathrm{y}$, and $\mathrm{z}$ directions, respectively. Furthermore, $C=\left\{C_{i} \mid i=1,2, \ldots, 10\right\}, C_{i}=\left(C_{i x}, C_{i y}, C_{i z}\right)$ denotes the 3D coordinates of the labeling identified in the $4 \mathrm{D}$ lung CT. There are 9 intervals $(\Delta t)$ when acquiring the $4 \mathrm{D}$ lung CT $I$. The $3 \mathrm{D}$ lung CT volume $I_{1}$ will match with the $3 \mathrm{D}$ coordinate $D_{j}$ by calculating the distance measure equation $F$. Thereby, the sequential correspondence between the 4D lung CT $I$ and the 10 coordinates of the labeling is constructed. Figure 1 shows the positions in the respiratory cycle for each 4D lung CT. 


\subsection{Image registration}

The image registration was used to measure the lungs' motion and deformation. Furthermore, the reference volume was nonrigidly registered separately to each of the 4D lung CT using a deformable registration method based on point-set-and-intensity [15]. The first registration step was to extract the point sets of the lung surface and vessels. Subsequently, the displacement vectors of these point sets were acquired through point set registration. Then, the sum of the squared Euclidean distance between the displacement vectors of these point sets and the displacement vectors based on the B-spline transformation model were minimized as a novel similarity measure to derive the rough transformation function. Finally, the rough transformation function was refined by the mutual information-based registration method. The B-spline transformation model that was used for the deformable registration was defined by a regular control point grid, with each control point having an associated 3D displacement.

This deformable registration method was chosen as it allows the transformation parameters themselves (the control point displacements) to be modeled rather than having to model the deformation field at each voxel. Furthermore, this registration method is particularly advantageous as it possesses a relatively high accuracy and is quickly conducted.

Prior to registrations, the lung was segmented by using an easy-to-implement automatic approach that is based on intensity and morphology.

\subsection{Model fitting}

\subsubsection{B-spline-based method}

An approximated B-spline was fitted to the registration results using a nonlinear least-squares optimization; in this process, the $\mathrm{x}, \mathrm{y}$, and $\mathrm{z}$ displacement for every registration control point was related to a respiratory cycle position [7]. The positions of the registration results (i.e., the 3D CTs) in the respiratory cycle were assigned by a phase-based approach. Although the entire transformation can be calculated by finding the displacement of every registration control point at the desired respiratory cycle position, it cannot be calculated by finding the displacement of every registration control point at any given value of the respiratory signal, which is hardly useful for surgical navigation.

\subsubsection{PCA-based method}

PCA is a method that is used to identify data patterns and then expresses the distinctness and

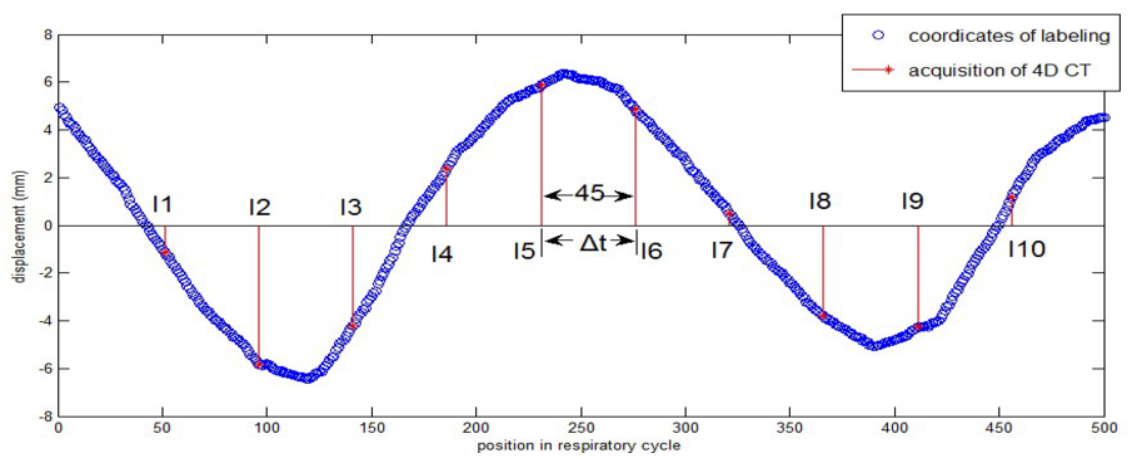

Fig. 1. The positions in the respiratory cycle for each 4D lung CT. 
similarities of this data. PCA can be used to compress this data through a dimensionality reduction while simultaneously minimizing the loss of information [6]. In the motion model building process, PCA is applied on both the internal motion that is described by the displacements of every registration control point as well as the corresponding external motion that is described by the amplitude of the external respiratory signal or respiratory phase. However, the respiratory phase is difficult to be calculated 'on-the-fly' and the amplitude of the external respiratory signal is not easily predicted. Given these methods' problems, a novel method using the original respiratory signal value was proposed.

\subsubsection{Proposed method}

In this study, the registration results of six 3D lung CT volumes $I=\left\{I_{i} \mid i=3,4, \ldots, 8\right\}$ within one respiratory cycle were adopted. An approximated B-spline was fitted to each $\mathrm{x}, \mathrm{y}$, and $\mathrm{z}$ displacement for every registration control point separately regarding time, which were obtained from the deformable registration of the 3D lung CT using a nonlinear least squares optimization [7]. The Bspline is similar to that used in the registration but is only one dimensional.

Using a nonlinear least squares optimization, a second-order Fourier series was fitted to the external respiratory signal in the anterior-posterior direction regarding time. The parameter R-square was set to 0.9990, and the root-mean-square error (RMSE) was set to $0.1241 \mathrm{~mm}$. Furthermore, a larger R-square value and a lower RMSE value are associated with a closer respiratory motion curve to the chosen function. This is consistent with the theoretical model proposed by Lujan, in which the respiratory motion curve is in line with the higher-order cosine function [16].

To obtain the corresponding control point displacements at any given respiratory signal value, a linear function was fitted for each of the control point displacements that define the B-spline transformation, thus relating it to the corresponding external respiratory signal value. Both the control point displacements as well as the corresponding respiratory signal value were sampled from the control point displacements fitting curves and the external respiratory signal fitting curve, respectively.

\section{Experiments and results}

To quantitatively evaluate the modeling's accuracy, the error between each landmark in the acquired and the corresponding model-generated CT volumes was calculated, which is then defined as the target modeling error (TME):
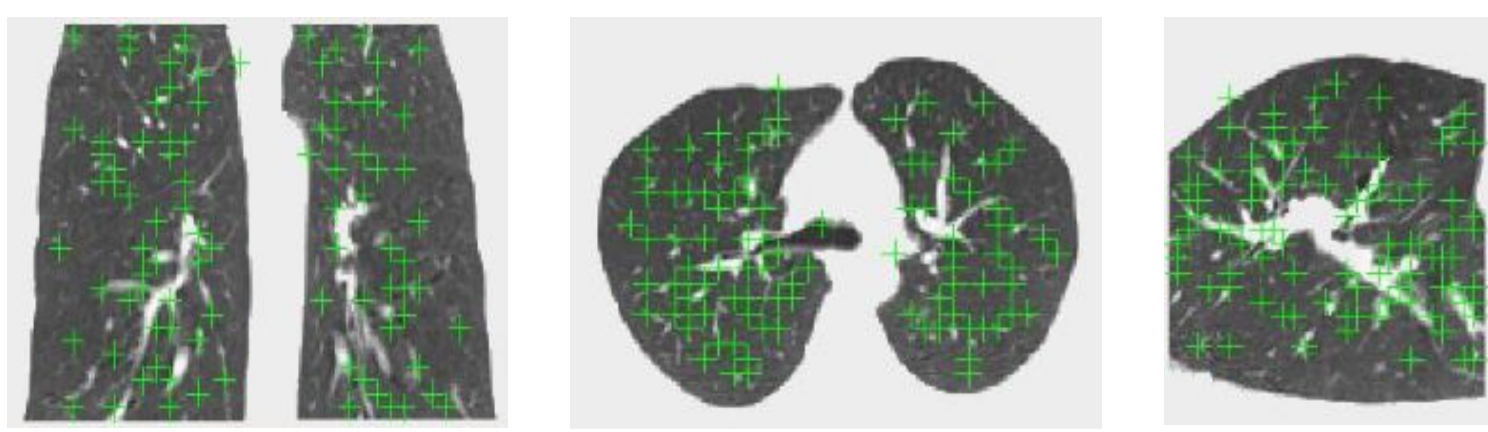

Fig. 2. Landmark locations projected onto the same slice in coronal, transverse, and sagittal planes. 
Table 1

Results from evaluating the motion models. The mean and the standard deviation (sd) are given for the target modeling error (TME)

\begin{tabular}{lllllll}
\hline & \multirow{2}{*}{ Type of surrogate signal $(\mathrm{s})$} & Voxel sizes $(\mathrm{mm})$ & \multicolumn{2}{l}{ Qty } & \multicolumn{3}{c}{ TME $(\mathrm{mm})$} \\
\cline { 5 - 7 } & & & acquired & used & mean & sd \\
\hline B-spline-based method & respiratory phase & $0.68 \times 0.68 \times 1.5$ & 20 & 20 & 2.15 & 0.50 \\
& respiratory phase & & & & 5.08 & 0.77 \\
PCA-based method & amplitude & $0.97 \times 0.97 \times 2.5$ & 20 & 10 & 3.63 & 0.42 \\
Proposed method & single signal & $0.677 \times 0.677 \times 0.5$ & 10 & 6 & 2.14 & 0.45 \\
\hline
\end{tabular}

$$
T M E=\frac{1}{m} \sum_{k=1}^{m} \sqrt{\left[0.677 \times\left(d_{x}^{k}-d_{R x}^{k}\right)\right]^{2}+\left[0.677 \times\left(d_{y}^{k}-d_{R y}^{k}\right)\right]^{2}+\left[0.500 \times\left(d_{z}^{k}-d_{R z}^{k}\right)\right]^{2}}
$$

where $d_{x}^{k}, d_{y}^{k}$, and $d_{z}^{k}$ are the corresponding $\mathrm{x}, \mathrm{y}$, and $\mathrm{z}$ coordinates of the $k$ th landmark in the acquired $\mathrm{CT}$, and $d_{R x}^{k}, d_{R y}^{k}$, and $d_{R z}^{k}$ are the corresponding $\mathrm{x}, \mathrm{y}$, and $\mathrm{z}$ coordinates of the $k$ th landmark in the model-generated CT. Furthermore, $m$ is the number of anatomical landmarks, which are generated from a semi-automatic landmark annotating system [17]. In this paper, 100 well-distributed corresponding landmarks were generated inside the lung domain of the reference 3D lung CT volume and the acquired CT volume that was to be estimated using the model. Figure 2 shows an example of landmark locations that are marked by green crosses in coronal, transverse, and sagittal planes of the reference 3D lung CT volume. The entire little green cross was projected onto the same slice.

The proposed model's TME is listed in Table 1 in millimeters. In addition, the type of surrogate signal(s), voxel sizes, the number of acquired 3D lung CT volumes (Qty) and used for constructing the motion model are also listed in Table 1.

For comparison, the modeling results (TME) that were obtained by using the B-spline-based method and those obtained by the PCA-based method are also given in Table 1.

From the results displayed in Table 1, the TME of the proposed method is commensurate with the TME of the B-spline-based method, whereas the proposed method requires $70 \%$ less 3D lung CTs. Compared to the PCA-based method that uses the phase of the external respiratory signal, the mean and sd of the TME of the proposed method decreased by $58 \%$ and $42 \%$, respectively. Similarly, compared to the PCA-based method that uses the amplitude of the external respiratory signal, the mean of the TME of the proposed method decreased $41 \%$ despite the $7 \%$ increase in the sd of the proposed method's TME. In general, when using similar amounts of 3D CT volumes, the mean of the TME of the proposed method is smaller than the mean of the PCA-based methods' TMEs, which indicates that the proposed method has a higher modeling accuracy. The above results indicate that the proposed method is successful in striking a balance between modeling accuracy and number of required 3D CT volumes. 


\section{Conclusion}

In this paper, a novel method for constructing a patient specific respiratory motion model with a low dose was proposed. The modeling's accuracy was quantitatively validated using landmarks, and the results of which suggest that the TME of the proposed method is commensurate with the TME of the B-spline-based method; however, the proposed method requires $70 \%$ less $3 \mathrm{D}$ lung CTs. In general, when using similar amounts of 3D CT volumes, the mean of the proposed method's TME is smaller than that of the PCA-based methods. Usually, the smaller the voxel size used in constructing the motion model, the smaller the mean TME of the proposed method is. In this study, the proposed method is again the superior method as the TME of the proposed method is commensurate with the TMEs of both the B-spline-based method as well as the PCA-based method, yet constructing the motion model requires the least number of acquired 3D lung CT volumes. Finally, the results indicate that the proposed method is successful in striking a balance between modeling accuracy and number of 3D CT, and therefore, it is a potentially useful tool for constructing a lung respiratory motion model with a low dose that will aid in accurately locating a lesion in surgery path planning and surgical navigation.

\section{Acknowledgement}

This work was supported by the National Natural Science Foundation of China (81371640), Project in Technology Program in Suzhou (SH201210), Special Project in Clinical Medicine of Jiangsu Province, China (BL2012049), and Development Project of Experimental Equipment, Chinese Academy of Sciences.

\section{References}

[1] S. Shimizu, H. Shirato, K. Kagei, T. Nishioka, X. Bo, H. Dosaka-Akita, S. Hashimoto, H. Aoyama, K. Tsuchiya and K. Miyasaka, Impact of respiratory movement on the computed tomographic images of small lung tumors in threedimensional (3D) radiotherapy, International Journal of Radiation Oncology Biology Physics 46 (2000), 1127-1133.

[2] S. Ahn, B. Yi, Y. Suh, J. Kim, S. Lee, S. Shin, S. Shin and E. Choi, A feasibility study on the prediction of tumour location in the lung from skin motion, The British Journal of Radiology 77 (2004), 588-596.

[3] J.R. McClelland, D.J. Hawkes, T. Schaeffter and A.P. King, Respiratory motion models: A review, Medical Image Analysis 17 (2012), 19-42.

[4] Y. Seppenwoolde, H. Shirato, K. Kitamura, S. Shimizu, M. van Herk, J.V. Lebesque and K. Miyasaka, Precise and realtime measurement of 3D tumor motion in lung due to breathing and heartbeat, measured during radiotherapy, International Journal of Radiation Oncology Biology Physics 53 (2002), 822-834.

[5] Y. Seppenwoolde, R.I. Berbeco, S. Nishioka, H. Shirato and B. Heijmen, Accuracy of tumor motion compensation algorithm from a robotic respiratory tracking system: A simulation study, Medical Physics 34 (2007), 2774-2784.

[6] H. Fayad, T. Pan, O. Pradier and D. Visvikis, Patient specific respiratory motion modeling using a 3D patient's external surface, Medical Physics 39 (2012), 3386-3395.

[7] J.R. McClelland, J.M. Blackall and S. Tarte, A continuous 4D motion model from multiple respiratory cycles for use in lung radiotherapy, Medical Physics 33 (2006), 3348-3358.

[8] K.H. Chang, M.C. Ho, C.C. Yeh, Y.C. Chen, F.L. Lian, J.Y. Yen and Y.Y. Chen, Effectiveness of external respiratory surrogates for in vivo liver motion estimation, Medical Physics 39 (2012), 5293-5301.

[9] B. Dong, Y.J. Graves, X. Jia and S.B. Jiang, Optimal surface marker locations for tumor motion estimation in lung cancer radiotherapy, Physics in Medicine and Biology 57 (2012), 8201-8215.

[10] T. Zhao, W. Lu, D. Yang, S. Mutic, C.E. Noel, P.J. Parikh, J.D. Bradley and D.A. Low, Characterization of free breathing patterns with 5D lung motion model, Medical Physics 36 (2009), 5183-5189. 
[11] J.D. Hoisak, K.E. Sixel, R. Tirona, P.C. Cheung and J.P. Pignol, Correlation of lung tumor motion with external surrogate indicators of respiration, International Journal of Radiation Oncology Biology Physics 60 (2004), 1298-1306.

[12] S.S. Vedam, V.R. Kini, P.J. Keall, V. Ramakrishnan, H. Mostafavi and R. Mohan, Quantifying the predictability of diaphragm motion during respiration with a noninvasive external marker, Medical Physics 30 (2003), 505-513.

[13] A.P. King, K.S. Rhode, R.S. Razavi and T.R. Schaeffter, An adaptive and predictive respiratory motion model for image-guided interventions: Theory and first clinical application, Medical Imaging 28 (2009), 2020-2032.

[14] R. Colgan, J. McClelland, D. McQuaid, P.M. Evans, D. Hawkes, J. Brock, D. Landau and S. Webb, Planning lung radiotherapy using 4D CT data and a motion model, Physics in Medicine and Biology 53 (2008), 5815-5830.

[15] W. Xia, X. Gao, and X. Wang, PSI: Point-Set-and-Intensity-Based algorithm for 3D CT deformable registration, Journal of Medical Imaging and Health Informatics 4 (2014), 444-447.

[16] A.E. Lujan, E.W. Larsen, J.M. Balter and R.K.T. Haken, A method for incorporating organ motion due to breathing into 3D dose calculations, Medical Physics 26 (1999), 715-720.

[17] K. Murphy, B. Ginneken, J.W. Pluim, S. Klein and M. Staring, Semi-automatic reference standard construction for quantitative evaluation of lung CT registration, in: Medical Image Computing and Computer-Assisted InterventionMICCAI 2008, D. Metaxas et al., eds., Springer Berlin Heidelberg, 2008, pp. 1006-1013. 